\title{
SGLT2 Inhibitor Treatment Outcome in Nonalcoholic Fatty Liver Disease Complicated with Diabetes Mellitus: The Long-term Effects on Clinical Features and Liver Histopathology
}

Norio Akuta ${ }^{1}$, Yusuke Kawamura ${ }^{1}$, Shunichiro Fujiyama ${ }^{1}$, Hitomi Sezaki ${ }^{1}$, Tetsuya Hosaka ${ }^{1}$, Masahiro Kobayashi ${ }^{1}$, Mariko Kobayashi ${ }^{2}$, Satoshi Saitoh ${ }^{1}$, Fumitaka Suzuki ${ }^{1}$, Yoshiyuki Suzuki ${ }^{1}$, Yasuji Arase ${ }^{1}$, Kenji Ikeda ${ }^{1}$ and Hiromitsu Kumada ${ }^{1}$

\begin{abstract}
:
Objective The aim of this study was to determine the long-term effects of a sodium-glucose cotransporter 2 inhibitor (SGLT2i) in nonalcoholic fatty liver disease (NAFLD) patients with type 2 diabetes mellitus (T2 DM) on the clinical features and liver histopathology.

Methods In this retrospective study, the long-term histological impacts of SGLT2i in NAFLD patients with T2DM were investigated.

Patients Seven patients with NAFLD and T2DM were treated for the long term with $100 \mathrm{mg} /$ day canagliflozin, an SGLT2i, and liver biopsies were obtained at the 3 points of pretreatment, 24 weeks, and $\geq 1$ year (third liver biopsy) after the start of treatment. Six of seven patients were evaluated with third liver biopsy at the point of three or more years. The primary outcome was liver histopathological changes (defined as a decrease in the NAFLD activity score of one point or more without worsening of the fibrosis stage, compared to pretreatment).

Results All 7 patients showed worsening of body mass index and waist circumference at the third liver biopsy compared to 24 weeks. However, the scores of steatosis, lobular inflammation, ballooning, and fibrosis stage improved at the third liver biopsy in $57 \%, 43 \%, 14 \%$, and $29 \%$ of the patients, respectively, compared to pretreatment. One of the seven patients showed histopathological worsening at the third liver biopsy compared to pretreatment, but the improvement was maintained in the other six patients.

Conclusion The long-term treatment of NAFLD complicated by T2DM using an SGLT2i is associated with long-term improvement in liver histopathology despite the worsening of clinical features.
\end{abstract}

Key words: nonalcoholic fatty liver disease, nonalcoholic steatohepatitis, diabetes mellitus, SGLT2 inhibitor, liver biopsy, long-term, hepatocyte steatosis, fibrosis stage

(Intern Med 59: 1931-1937, 2020)

(DOI: 10.2169/internalmedicine.4398-19)

\section{Introduction}

Nonalcoholic fatty liver disease (NAFLD) is the most common liver disease worldwide (1-4) and encompasses a wide spectrum of pathologies, ranging from benign nonalco- holic fatty liver to nonalcoholic steatohepatitis (NASH). Thus, NAFLD can progress to liver cirrhosis, hepatocellular carcinoma (HCC), or liver failure, even in the absence of excessive alcohol intake (5).

Sodium-glucose cotransporter 2 inhibitor (SGLT2i) improves the outcome of patients with type 2 diabetes mellitus

${ }^{1}$ Department of Hepatology, Toranomon Hospital and Okinaka Memorial Institute for Medical Research, Japan and ${ }^{2}$ Liver Research Laboratory, Toranomon Hospital, Japan

Received: December 29, 2019; Accepted: March 30, 2020; Advance Publication by J-STAGE: May 23, 2020

Correspondence to Dr. Norio Akuta, akuta-gi@umin.ac.jp 
(T2DM) by enhancing urinary glucose excretion, and reducing the risk of cardiovascular and renal complications $(6,7)$. We also reported recently that an SGLT2i improved the histopathological findings in NAFLD complicated by T2DM by reducing the scores of steatosis, lobular inflammation, ballooning, and fibrosis stage at 24 weeks compared to pretreatment scores [with histopathological improvement taken as a $\geq 1$-point reduction in the NAFLD activity score (NAS) without worsening of the fibrosis stage] $(8,9)$. Similar results using other SGLT2is were also reported by another group (significant improvements at 24 weeks in steatosis, ballooning and fibrosis] (10), highlighting the therapeutic potential of SGLT2is as effective agents against NAFLD complicated with T2DM.

The aim of the present retrospective study was to determine the long-term impact of $100 \mathrm{mg} /$ day canagliflozin, an SGLT2i, on the clinical features and liver histopathology of NAFLD patients complicated with T2DM.

\section{Materials and Methods}

\section{Study design and participants}

In this retrospective study, 7 Japanese patients with NAFLD and T2DM received long-term treatment with 100 mg canagliflozin/day at our hospital between November 2015 and December 2019, and the clinical and histopathological effects (e.g., steatosis, lobular inflammation, ballooning, and fibrosis stage) of such long-term treatment were assessed at 3 points: pretreatment (baseline, first liver biopsy), 24 weeks (second liver biopsy), and $\geq 1$ year (third liver biopsy) after the start of treatment. Six of the seven patients were evaluated with a third liver biopsy at three or more years. The results of the first and second liver biopsies were based on our previous prospective clinical trial UMIN 000018166 (https://upload.umin.ac.jp/cgi-open-bin/ctr/index. cgi) $(8,9)$. Patients with histopathological changes of steatosis in at least $5 \%$ of hepatocytes and those with a history of alcohol intake of $<20 \mathrm{~g} /$ day were included in the analysis. None of the selected patients had other underlying liver disease (e.g., viral hepatitis, autoimmune hepatitis, druginduced liver disease, or primary biliary cholangitis), systemic autoimmune diseases (e.g., systemic lupus erythematosus or rheumatoid arthritis), or metabolic diseases known to affect the liver (e.g., hemochromatosis, $\alpha$-1-antitrypsin deficiency, or Wilson's disease).

The study protocol was approved by the Toranomon Hospital institutional review board (\#953). The study was conducted in compliance with the International Conference on Harmonisation Guideline for Good Clinical Practice (E6) and the 2013 Declaration of Helsinki. Written informed consent for the liver biopsy was provided by all patients.

\section{Liver histopathology}

Liver biopsy specimens were obtained using a 14-gauge modified Vim Silverman needle (Tohoku University style;
Kakinuma Factory, Tokyo, Japan), a 16-gauge core tissue biopsy needle (Bard Peripheral Vascular, Tempe, USA), or surgical resection. The minimum acceptable size of the liver biopsy specimen was set at $>1.5 \mathrm{~cm}$ and/or containing more than 11 portal tracts. The specimen was fixed in $10 \%$ formalin and cut into sections, which were then stained with hematoxylin-eosin, Masson trichrome, silver impregnation, or periodic acid-Schiff after diastase digestion. Four pathologists (K.K., F.K., T.F., and T.F.) who were blinded to the clinical findings evaluated each of the specimens, and the final assessment was reached by consensus.

Steatosis grades $0,1,2$, and 3 corresponded to steatosis of hepatocytes of $<5 \%, \geq 5 \%$ to $<33 \%, \geq 33 \%$ to $<66 \%$, and $\geq 66 \%$ of the total, respectively. Lobular inflammation scores of $0,1,2$, and 3 represented no inflammatory foci, $<2$ foci, 2-4 foci, and $\geq 4$ foci per $200 \times$ field, respectively. Hepatocyte ballooning of none, few, and many cells was scored as 0,1 , and 2, respectively. NAS represented the sum of scores of steatosis, lobular inflammation, and hepatocyte ballooning (range, 0-8 points) (11). The fibrosis stage was classified as $0,1,2,3$, and 4 using the criteria defined previously $(11,12)$. NASH was defined according to the Fatty Liver: Inhibition of Progression (FLIP) algorithm (13).

\section{Outcomes}

The primary outcome measure included histopathological changes in the individual histological components of NASH from baseline (first liver biopsy) to 24 weeks (second liver biopsy) and $\geq 1$ year (third liver biopsy) after the start of treatment. Histopathological improvement was defined as a decrease in the NAS of one point or more without worsening of the fibrosis stage. The secondary outcomes included changes in clinical parameters (including clinical features, laboratory test results, and imaging findings) from baseline to 24 weeks and $\geq 1$ year after the start of treatment. We analyzed the clinicopathological parameters that could affect the NAFLD prognosis using the Fib-4 index with the following formula: [age (year) $\times$ AST (aspartate aminotransferase) $(\mathrm{U} / \mathrm{L})] /\left[\right.$ platelet count $\left(10^{9} / \mathrm{L}\right) \times \sqrt{\text { alanine aminotrans- }}$ ferase (ALT) (U/L)], which has been used previously to assess the progression of liver fibrosis (14).

\section{Statistical analyses}

Wilcoxon's test was used to compare paired samples. All $\mathrm{p}$ values less than 0.05 by the two-tailed test were considered significant. Statistical analyses were performed using the SPSS software program (SPSS, Chicago, USA).

\section{Results}

\section{Histopathological changes}

Table and Fig. 1 summarize the changes in histopathological scores at the time of the three liver biopsies. The results of the first two liver biopsies have already been reported in our previous prospective study $(8,9)$. Case 2 dis- 
Table. Histological Findings at the Time of Liver Biopsies.

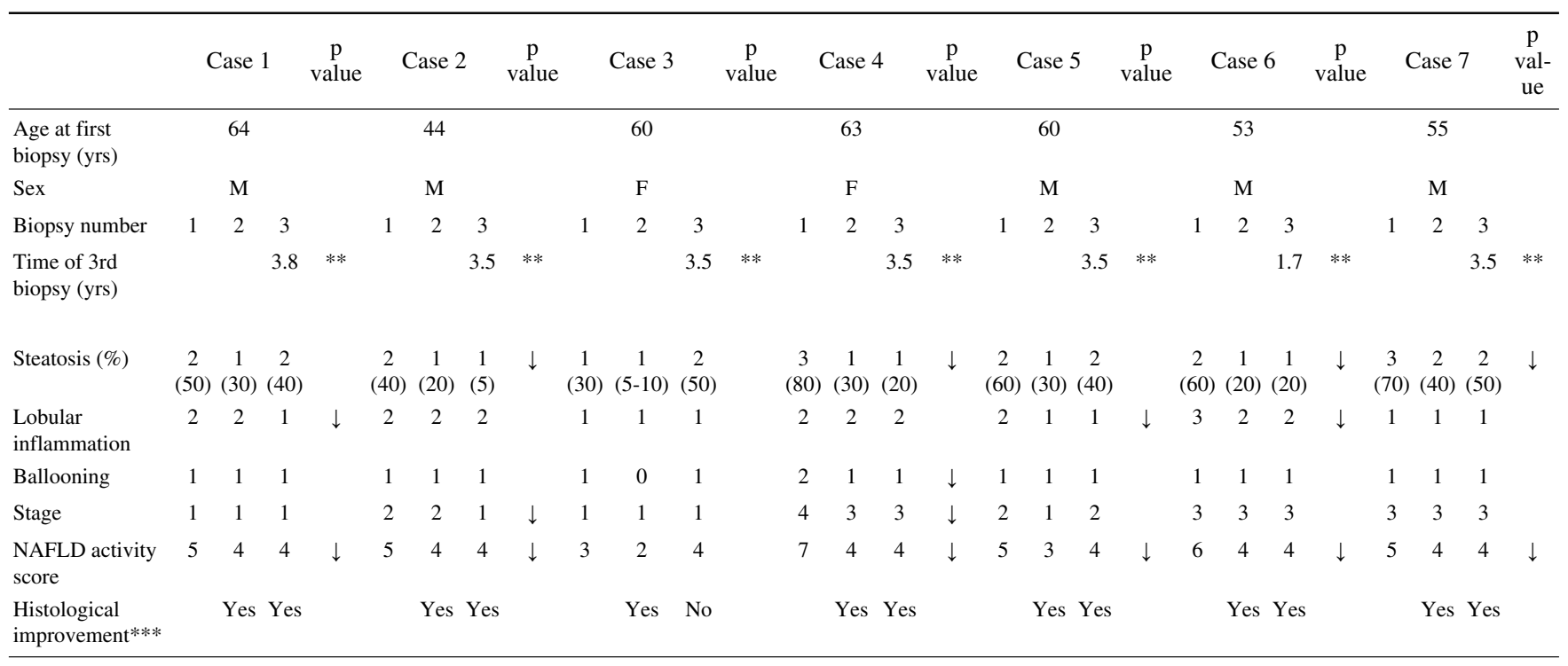

Diagnosis was based on the FLIP algorithm.

The first and second biopsies were conducted before treatment and at 24 weeks, respectively in all patients.

The diagnosis was confirmed to be NASH in all patients and all biopsies, except as non-NASH in biopsy 2 of Case 3.

*Results of 1st and 2nd liver biopsies, are based on the previous prospective study (Reference 8,9).

**Factors that tended to decrease at 3rd biopsy relative to 1st biopsy, are indicated by black arrow.

***Histological improvement; a decrease in NAS of one point or more without worsening in fibrosis stage, relative to the baseline (pretreatment).

NAFLD: nonalcoholic fatty liver disease, M: male, F: female, FLIP: Fatty Liver Inhibition of Progossion , NASH: nonalcoholic steatohepatitis, NAS: NAFLD activity score

continued medication of SGLT2i by self-judgment for one year between second and third liver biopsies, but the other six patients continued SGLT2i treatment until the third liver biopsy. The scores of steatosis, lobular inflammation, ballooning, and fibrosis stage improved in $86 \%$ (Cases 1, 2, 4, 5, 6, 7), 29\% (Cases 5, 6), 29\% (Cases 3, 4), and 29\% (Cases 4,5$)$ at the second liver biopsy, compared to pretreatment, respectively. The results showed that in all 7 patients, treatment with $100 \mathrm{mg}$ canagliflozin/day for 24 weeks was associated with histopathological improvement.

Analyses of the tissue samples obtained from the 7 patients at $\geq 1$ year after starting treatment (third liver biopsy) showed improvements in the scores of steatosis, lobular inflammation, ballooning, and fibrosis stage in 57\% (Cases 2, 4, 6, 7), 43\% (Cases 1, 5, 6), 14\% (Cases 4), and 29\% (Cases 2, 4), compared to pretreatment, respectively. One (Case 3) of seven patients showed histopathological worsening at the third liver biopsy compared to pretreatment, but the improvement was maintained at the third biopsy in the other six patients. Representative pathological images of a worsening case (Case 3) and improved case (Case 4) at the third liver biopsy, compared to pretreatment, are shown in Figs. 2 and 3, respectively.

\section{Changes in clinical parameters}

Supplementary materials 1 and 2 summarize the changes in clinical parameters at the time of the three liver biopsies. The results of the first two liver biopsies have already been reported in our previous prospective study $(8,9)$. In all 7 patients, 24-week treatment with SGLT2i was associated with significant improvement in the clinical features, body mass index, waist circumference, serum aspartate aminotransferase, serum alanine aminotransferase, estimate glomerular filtration rate, fasting plasma glucose, HbA1c, HOMA-IR, type IV collagen 7S, serum ferritin, and imaging findings (transient elastography and magnetic resonance spectroscopy) compared to pretreatment values.

Surprisingly, all 7 patients showed worsening of secondary outcome measures, including the body mass index and waist circumference, after $\geq 3$ years of treatment, compared to 24 weeks (body mass index, $\mathrm{p}=0.018$; and waist circumference, $\mathrm{p}=0.018$ ) (Fig. 4). Parameters of glucose metabolism were also deteriorated compared with the values after 24-week treatment (fasting plasma glucose, $\mathrm{p}=0.018$; and HbA1c, p=0.018) (Fig. 4).

\section{Discussion}

We reported recently a favorable outcome following 24week treatment with SGLT2i with respect to the histopathological features of NAFLD complicated by T2DM (8-10). The present study was an extension of the above studies, and the results showed that long-term treatment with SGLT2 i was associated with long-term liver histopathological improvement (at $\geq 3$ years in 6 of 7 patients), despite worsening of clinical features. The difference between the primary and secondary outcomes might be due, at least in part, to differences in the effects of SGLT2i on visceral adipose tissue and subcutaneous adipose tissue. Previous studies have shown that weight loss associated with SGLT2i treatment was mainly due to the loss of fat mass rather than lean mass, and a computed tomography analysis of abdominal fat 


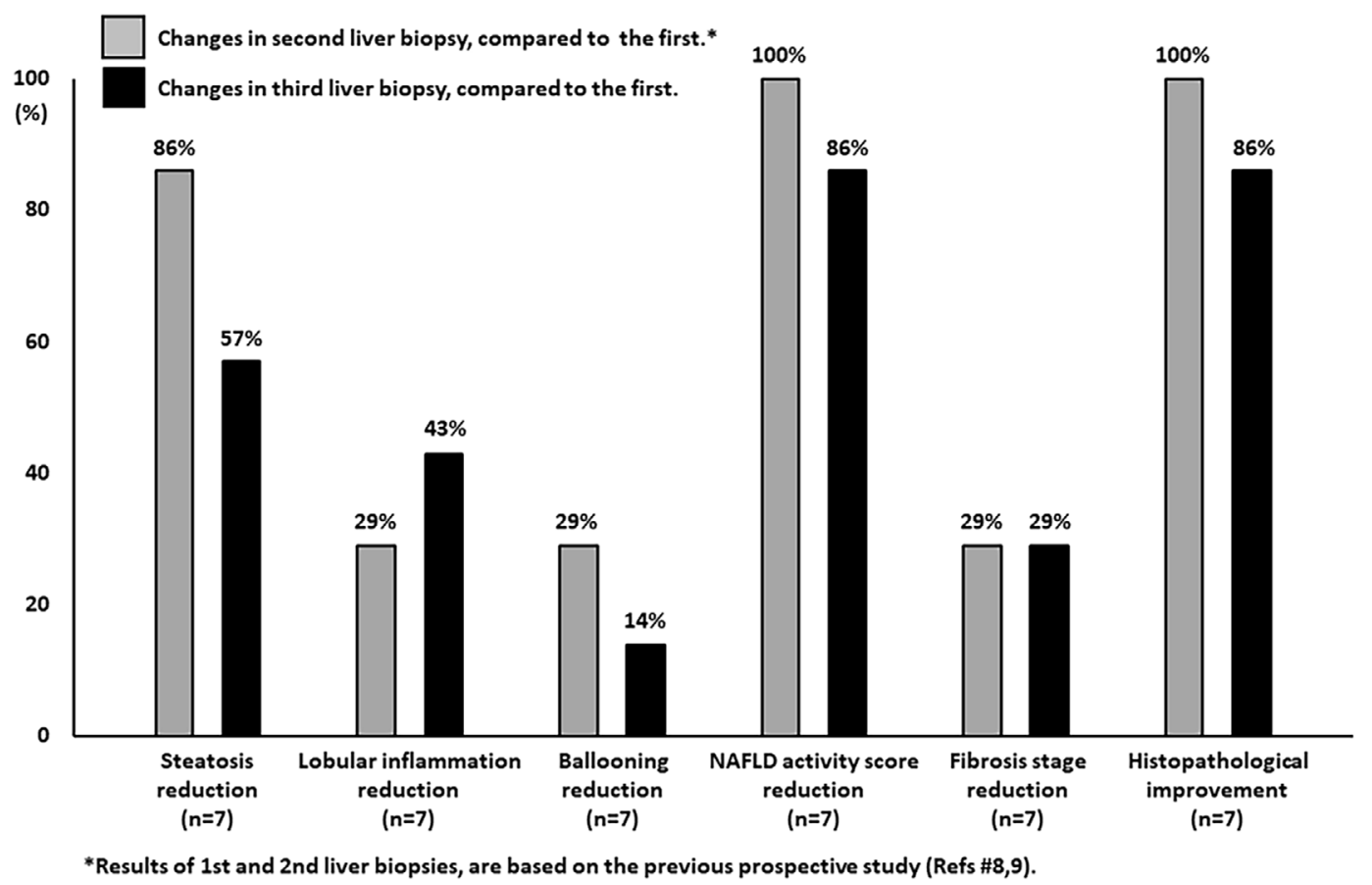

Figure 1. Changes from baseline (first liver biopsy) to 24 weeks (second liver biopsy) and $\geq 1$ year (third liver biopsy) after the start of treatment with SGLT2 inhibitor in individual histopathological components of NASH. Six of 7 patients were evaluated by a third liver biopsy at $\geq 3$ years. Histopathological improvement was defined as a decrease in the NAFLD activity score of one point or more without worsening of the fibrosis stage.

in patients treated with SGLT2i showed a slightly greater reduction in visceral adipose tissue than in subcutaneous adipose tissue (15).

The study of Ferrannini et al. (16) in patients with chronic glycosuria demonstrated that long-term treatment with SGLT2i (90 weeks) elicited an adaptive increase in energy intake, and the combination of SGLT2i with calorie restriction resulted, in at least some patients, in major weight loss (16). Thus, calorie restriction should be applied to prevent worsening of clinical features and glucose metabolism and possibly to prevent histopathological worsening (e.g., Case 3).

Previous reports suggested that SGLT2i might improve fibrosis stage in NAFLD patients (8-10), but the mechanism underlying the improvement in the fibrosis stage is still unclear. Higher levels of HOMA-IR, as a surrogate marker of insulin resistance, are a risk factor for severe fibrosis of NAFLD $(17,18)$. The present study indicated that the levels of HOMA-IR were significantly improved at the second and third liver biopsies compared to the pretreatment levels, and the improvement was maintained at the third biopsy (Supplementary material 1 and 2). However, we must be careful when interpreting the present findings, as only two patients showed improvement in the fibrosis stage. Hence, further studies based on a large number of patients should be performed to investigate the association between insulin resistance and fibrosis stage.

The long-term effects of SGLT2i on liver-related events in NAFLD patients with T2DM remain unknown. In two clini- cal trials involving patients with T2DM at high risk of cardiovascular disease (the CANVAS Program and EMPA-REG OUTCOME), patients treated with SGLT2i showed a lower risk of cardiovascular events than those who received placebo $(6,7)$. The results also showed the possible benefits of SGLT2 $i$ with respect to the progression of albuminuria and the composite outcome of a sustained $40 \%$ reduction in the estimated glomerular filtration rate, the need for renalreplacement therapy, or death from renal causes (7). Fibrosis stage was reported to be an independent and significant predictor of the overall mortality and liver-related events in NAFLD patients (19). The present study indicated that the scores of fibrosis stage improved in $29 \%$ of cases at the second and third liver biopsy, compared to pretreatment. Improvement in the fibrosis stage with SGLT2i might therefore lead to a good prognosis of NAFLD. Further large-scale long-term follow-up studies are needed to investigate the effects of SGLT2i on liver-related events.

In conclusion, we demonstrated in the present study the potential long-term effectiveness of SGLT2i in NAFLD patients complicated with T2DM. This retrospective study has several limitations. First, the study design was an uncontrolled, before-after study based on a small number of patients. Second, there were two patients (Case 3 and 5) who had other additional antidiabetic drugs introduced between the second and third liver biopsies, although no other additional antidiabetic drugs with a high evidence level for NAFLD [e.g., Pioglitazone (20) and Glucagon-like peptide-1 receptor agonists (21)] were introduced. Further prospective 
(A) Hematoxylin-eosin staining, $100 \times$
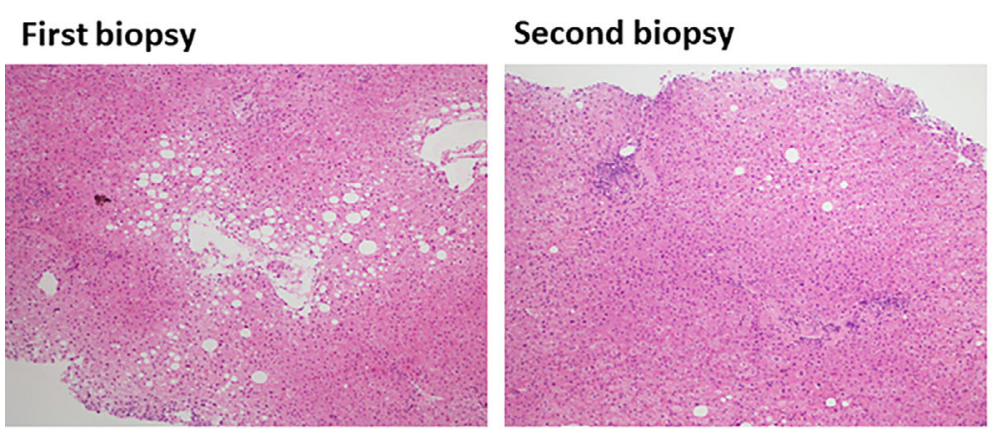

Third biopsy

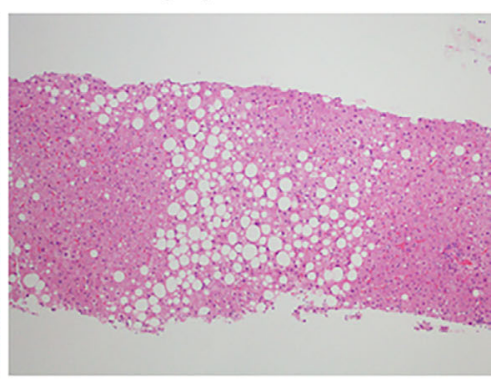

(B) Masson trichrome staining, $100 \times$
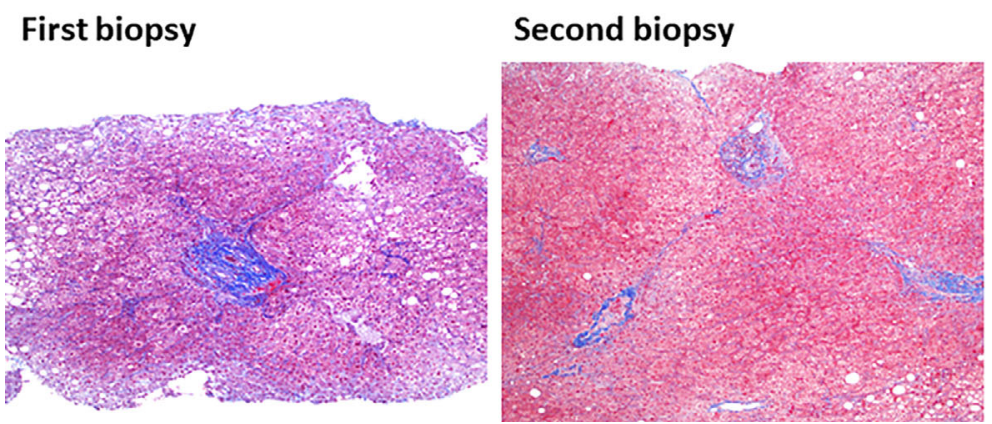

\section{Third biopsy}

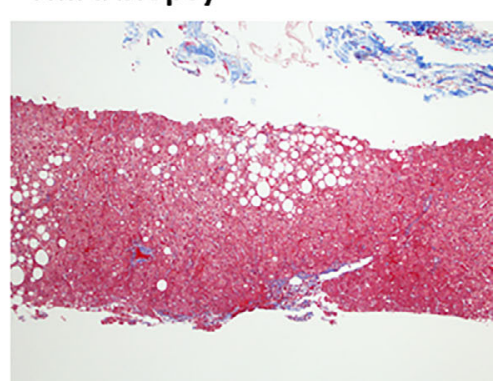

Figure 2. Representative pathological images of a worsening case (Case 3 ) at the third liver biopsy compared to pretreatment are shown. Histological changes at the three points of pretreatment (first liver biopsy), 24 weeks (second liver biopsy), and 3.5 years (third liver biopsy) after the start of SGLT2 inhibitor. (A) Hematoxylin-eosin staining, 100×. (B) Masson trichrome staining, 100×.

(A) Hematoxylin-eosin staining, $100 \times$
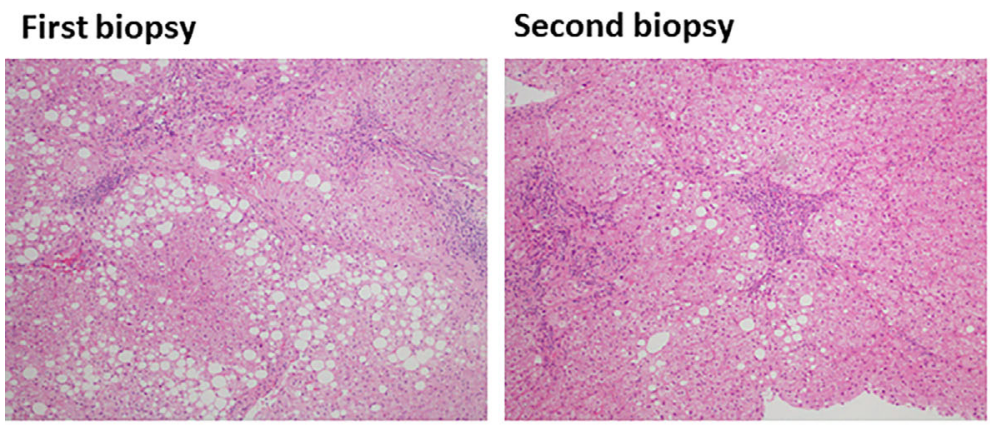

\section{Third biopsy}

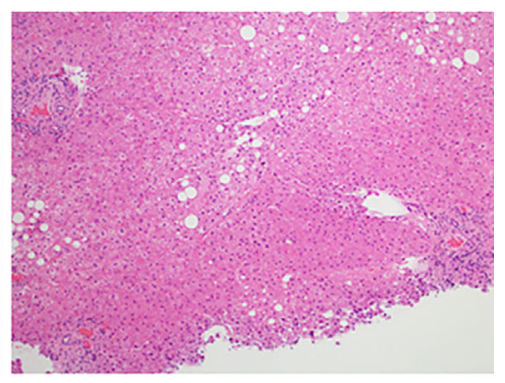

(B) Masson trichrome staining, $100 \times$

\section{First biopsy}

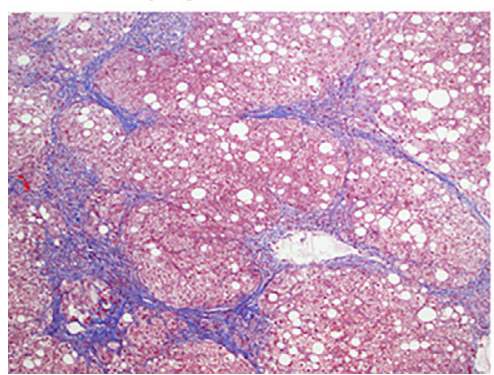

Second biopsy

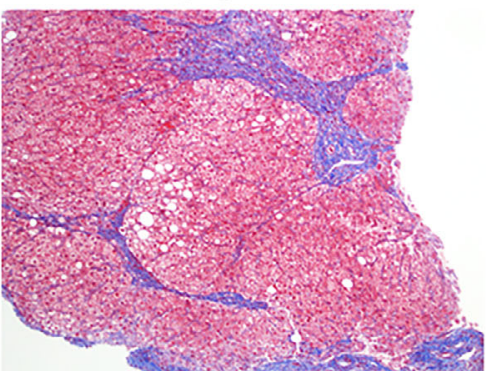

\section{Third biopsy}

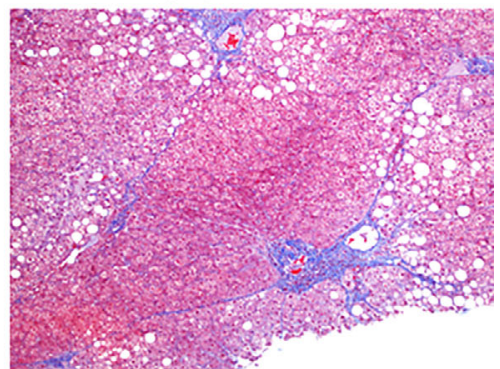

Figure 3. Representative pathological images of an improved case (Case 4) at the third liver biopsy compared to pretreatment are shown. Histological changes at the three points of pretreatment (first liver biopsy), 24 weeks (second liver biopsy), and 3.5 years (third liver biopsy) after the start of SGLT2 inhibitor. (A) Hematoxylin and Eosin staining, 100×. (B) Masson trichrome staining, 100×. 


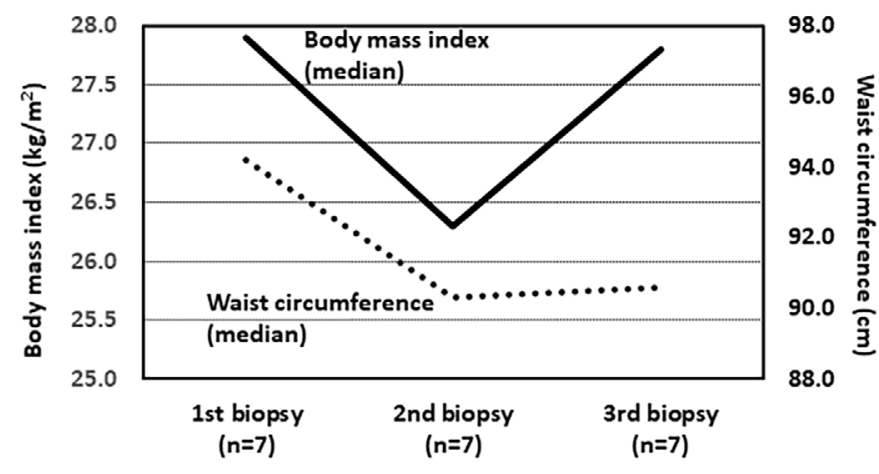

Body mass index

1st vs. 2nd. $P=0.018$ *

2nd vs. 3rd. $P=0.018$

1st vs. 3 rd. $P=0.063$

Waist circumference

1st vs. 2 nd. $P=0.018^{*}$

2nd vs. 3rd. $P=0.018$

1st vs. 3rd. $P=0.612$

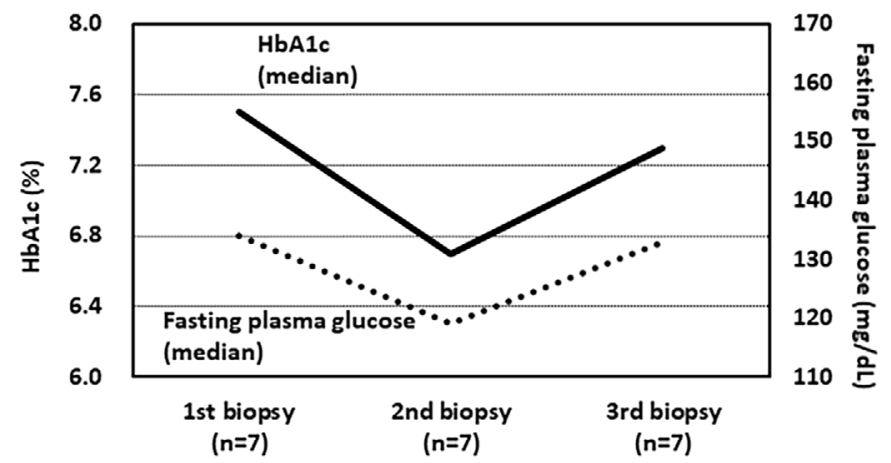

HbA1c

1st vs. 2nd. $P=0.043^{*}$

2nd vs. 3rd. $P=0.018$

1st vs. 3rd. $P=0.611$

Fasting plasma glucose

1st vs. 2nd. $P=0.018^{*}$

2nd vs. 3rd. $P=0.018$

1st vs. 3rd. $P=0.176$

*Results at the time of first and second liver biopsies, are based on our previous prospective study (references 8,9 ).

Figure 4. Serial changes in the median values of clinical parameters during the study. Note the significant worsening at the third liver biopsy (body mass index, second vs. third, $p=0.018$; waist circumference, second vs. third, $\mathrm{p}=\mathbf{0 . 0 1 8}$ ). Significant worsening of glucose metabolism was noted at the third liver biopsy (fasting plasma glucose, second vs. third, p=0.018; HbA1c, second vs. third, p=0.018).

studies in large population samples are needed to confirm the long-term effects of SGLT2i on the histopathological features of NAFLD.

The study protocol was approved by the Human Ethics Review Committee at Toranomon Hospital, and each patient gave their signed informed consent at the time of the liver histological diagnosis. The study was conducted in compliance with the International Conference on Harmonization Guideline for Good Clinical Practice (E6) and the 2013 Declaration of Helsinki.

Author's disclosure of potential Conflicts of Interest (COI). Norio Akuta: Honoraria, Mitsubishi Tanabe Pharma, AbbVie and Gilead Sciences. Masahiro Kobayashi: Honoraria, Eisai. Yasuji Arase: Honoraria, AbbVie. Hiromitsu Kumada: Honoraria, MSD, Gilead Sciences, AbbVie, Eisai and Dainippon Sumitomo Pharma.

\section{Financial Support}

This study was supported in part by a Grant-in-Aid from the Japan Agency for Medical Research and Development (\#JP19fk 0210040) (Norio Akuta) and a Grant-in-Aid for Scientific Research and Development from the Ministry of Health, Labour and Welfare of Japan (\#19HC1001) (Norio Akuta).

\section{Acknowledgement}

We thank the following individuals for their assistance in histopathological diagnosis: Keiichi Kinowaki, M.D. and Takeshi Fujii, M.D., Department of Pathology, Toranomon Hospital; Fukuo Kondo, M.D. and Toshio Fukusato, M.D., Department of Pathology, Teikyo University School of Medicine.

\section{References}

1. Angulo P. Nonalcoholic fatty liver disease. N Engl J Med 346: 1221-1231, 2002.

2. Williams R. Global changes in liver disease. Hepatology 44: 521526, 2006.

3. Sumida Y, Nakajima A, Itoh Y. Limitations of liver biopsy and non-invasive diagnostic tests for the diagnosis of nonalcoholic fatty liver disease/nonalcoholic steatohepatitis. World J Gastroenterol 20: 475-485, 2014.

4. Akuta N, Kawamura Y, Arase Y, et al. Hepatocellular carcinoma is the most common liver-related complication in patients with histopathologically-confirmed NAFLD in Japan. BMC Gastroenterol 18: 165, 2018.

5. Kleiner DE, Brunt EM. Nonalcoholic fatty liver disease: pathologic patterns and biopsy evaluation in clinical research. Semin Liver Dis 32: 3-13, 2012.

6. Zinman B, Wanner C, Lachin JM, et al. Empagliflozin, cardiovascular outcomes, and mortality in type 2 diabetes. $\mathrm{N}$ Engl $\mathrm{J}$ Med 373: 2117-2128, 2015.

7. Neal B, Perkovic V, Mahaffey KW, et al. Canagliflozin and cardiovascular and renal events in type 2 diabetes. $\mathrm{N}$ Engl $\mathrm{J}$ Med 377: 644-657, 2017.

8. Akuta N, Watanabe C, Kawamura Y, et al. Effects of a sodiumglucose cotransporter 2 inhibitor in nonalcoholic fatty liver disease 
complicated by diabetes mellitus: preliminary prospective study based on serial liver biopsies. Hepatol Commun 1: 46-52, 2017.

9. Akuta N, Kawamura Y, Watanabe C, et al. Impact of sodium glucose cotransporter 2 inhibitor on histological features and glucose metabolism of non-alcoholic fatty liver disease complicated by diabetes mellitus. Hepatol Res 49: 531-539, 2019.

10. Lai LL, Vethakkan SR, Nik Mustapha NR, et al. Empagliflozin for the treatment of nonalcoholic steatohepatitis in patients with type 2 diabetes mellitus. Dig Dis Sci 65: 623-631, 2020.

11. Kleiner DE, Brunt EM, Van Natta M, et al. Design and validation of a histological scoring system for nonalcoholic fatty liver disease. Hepatology 41: 1313-1321, 2005.

12. Brunt EM, Janney CG, Di Bisceglie AM, et al. Nonalcoholic steatohepatitis: a proposal for grading and staging the histological lesions. Am J Gastroenterol 94: 2467-2474, 1999.

13. Bedossa P. Utility and appropriateness of the fatty liver inhibition of progression (FLIP) algorithm and steatosis, activity, and fibrosis (SAF) score in the evaluation of biopsies of nonalcoholic fatty liver disease. Hepatology 60: 565-575, 2014.

14. Sterling RK, Lissen E, Clumeck N, et al. Development of a simple noninvasive index to predict significant fibrosis in patients with HIV/HCV coinfection. Hepatology 43: 1317-1325, 2006.

15. Cefalu WT, Leiter LA, Yoon KH, et al. Efficacy and safety of canagliflozin versus glimepiride in patients with type 2 diabetes inadequately controlled with metformin (CANTATA-SU): 52 week results from a randomised, double-blind, phase 3 non-inferiority trial. Lancet 382: 941-950, 2013.
16. Ferrannini G, Hach T, Crowe $S$, et al. Energy balance after sodium-glucose cotransporter 2 inhibition. Diabetes Care 38: 1730-1735, 2015.

17. Sorrentino P, Terracciano L, D'Angelo S, et al. Predicting fibrosis worsening in obese patients with NASH through parenchymal fibronectin, HOMA-IR, and hypertension. Am J Gastroenterol 105: 336-344, 2010.

18. Fujii $\mathrm{H}$, Imajo $\mathrm{K}$, Yoneda $\mathrm{M}$, et al. HOMA-IR: an independent predictor of advanced liver fibrosis in nondiabetic non-alcoholic fatty liver disease. J Gastroenterol Hepatol 34: 1390-1395, 2019.

19. Akuta N, Kawamura Y, Arase Y, et al. Circulating microRNA-122 and fibrosis stage predict mortality of Japanese patients with histopathologically confirmed NAFLD. Hepatol Commun 4: 66-76, 2019.

20. Sanyal AJ, Chalasani N, Kowdley KV, et al. Pioglitazone, vitamin E, or placebo for nonalcoholic steatohepatitis. N Engl J Med 362: 1675-1685, 2010.

21. Armstrong MJ, Gaunt P, Aithal GP, et al. Liraglutide safety and efficacy in patients with non-alcoholic steatohepatitis (LEAN): a multicentre, double-blind, randomised, placebo-controlled phase 2 study. Lancet 387: 679-690, 2016.

The Internal Medicine is an Open Access journal distributed under the Creative Commons Attribution-NonCommercial-NoDerivatives 4.0 International License. To view the details of this license, please visit (https://creativecommons.org/licenses/ by-nc-nd/4.0/).

(C) 2020 The Japanese Society of Internal Medicine

Intern Med 59: 1931-1937, 2020 